\title{
Kernos
}

Revue internationale et pluridisciplinaire de religion grecque antique

$23 \mid 2010$

Varia

\section{Monique MUND-DOPCHIE, Ultima Thulé. Histoire d'un lieu et genèse d'un mythe}

\section{Jean-Michel Renaud}

\section{OpenEdition \\ Journals}

\section{Édition électronique}

URL : http://journals.openedition.org/kernos/1661

DOI : 10.4000/kernos. 1661

ISSN : 2034-7871

\section{Éditeur}

Centre international d'étude de la religion grecque antique

\section{Édition imprimée}

Date de publication : 1 janvier 2010

Pagination : 407-409

ISSN : 0776-3824

\section{Référence électronique}

Jean-Michel Renaud, «Monique mund-dopchie, Ultima Thulé. Histoire d'un lieu et genèse d'un mythe », Kernos [En ligne], 23 | 2010, mis en ligne le 15 septembre 2011, consulté le 21 septembre 2020. URL: http://journals.openedition.org/kernos/1661; DOI : https://doi.org/10.4000/kernos.1661 


\title{
Monique MUND-DOPCHIE, Ultima Thulé. Histoire d'un lieu et genèse d'un mythe
}

\author{
Jean-Michel Renaud
}

\section{RÉFÉRENCE}

Monique MUND-DOPCHIE, Ultima Thulé. Histoire d'un lieu et genèse d'un mythe, Genève, Droz, 2009. 1 vol. 15,5 × $22 \mathrm{~cm}, 494$ p. (Histoire des idées et critique littéraire, 449). ISBN : 978-2-600-01234-8.

1 L'A. est connue pour de nombreux travaux de valeur, qui suivent certains mythes dans leurs divers développements depuis l'Antiquité jusqu'à nos jours, ce qui constitue la vraie position humaniste. Le présent ouvrage nous offre une remarquable et copieuse étude (en quatre parties, de longueur variable : l'Antiquité, le Moyen Âge, les XVI ${ }^{\mathrm{e}}-\mathrm{XVII}^{\mathrm{e}}$ s. et $d u x{ }^{\prime}{ }^{e}$ s. à nos jours, avec chaque fois une brève conclusion) des diverses et nombreuses opinions qui ont été professées sur Thulé, depuis qu'elle a été mentionnée pour la première fois par le voyageur grec Pythéas de Marseille ( $\mathrm{IV}^{\mathrm{e}} \mathrm{s}$. av. J.-C. ?). Malheureusement, hormis quelques fragments en nombre limité, l'œuvre de Pythéas et celle de plusieurs auteurs relativement proches de son époque ont été perdues, ce qui limite l'information à des sources grecques et latines assez tardives, que l'A. examine d'une manière critique et prudente. Dès l'Antiquité, des auteurs ont tenté de situer Thulé, avec des identifications diverses, en tenant compte des réalités géographiques connues. À côté de cette démarche à tendance géographique, Thulé a aussi suscité l'imagination des littérateurs et le thème a rejoint des conceptions exprimées dans la Nékuya de l'Odyssée, dans divers tableaux du monde des morts et même dans l'Atlantide évoquée par Platon.

2 Après l'Antiquité, l'A. passe au Moyen Âge, où les sources sont peu nombreuses, mais où la recherche sur la situation de Thulé continue la tradition de l'Antiquité; elle se précise 
d'autant plus que, parmi les auteurs qui en parlent, plusieurs résidaient en Angleterre (Bède le Vénérable), dans le nord de l'Allemagne (comme Adam de Brème) ou en Scandinavie. À côté de données géographiques reprises à l'Antiquité ou inspirées par l'expérience géographique, le mythe de Thulé s'est aussi inséré dans une tradition littéraire qui subit l'influence celtique et finit par s'inscrire dans la matière de Bretagne.

$3 \quad{\text { Aux } \mathrm{XVI}^{\mathrm{e}} \text { et } \mathrm{XVII}}^{\mathrm{e}} \mathrm{s}$, , avec la Renaissance, la connaissance des textes antiques, grecs notamment, est meilleure et les publications relatives au sujet deviennent très nombreuses. Deux orientations se présentent: les efforts pour situer Thulé dans un cadre géographique et les développements dus à l'imagination autour de Thulé. En ce qui concerne le premier point, on se fonde sur les données des Anciens, sur les connaissances géographiques du temps et des descriptions de voyageurs (Christophe Colomb a prétendu être allé jusqu'à Thulé). À des considérations qui intéressent surtout les gens savants viennent s'ajouter des options liées à la politique : certains pays, dont la Suède, veulent contenir Thulé ou bien en être proches, afin de se donner un passé prestigieux. Tantôt Thulé serait une île inexistante dans la réalité, tantôt elle est située en Islande, ou bien dans le nord des îles britanniques (dans les Hébrides ou les Shetlands) ou bien en Scandinavie. Les spécialistes s'opposent, parfois dans des controverses vigoureuses, pour défendre la position qu'ils ont choisie et leur option peut avoir une influence sur les commentaires qu'ils font des textes antiques. L'autre hypothèse voit surtout dans Thulé un point de départ riche de symboles et de développements plus ou moins oniriques.

Dans la suite de conceptions très anciennes, notamment celtiques, conceptions christianisées, on a vu dans Thulé un séjour des morts, les glaces du nord et le feu du volcan Hekla servant à la punition des âmes condamnées au purgatoire ou à l'enfer. Située aux confins du monde, dans une région glaciale, Thulé est remarquable dans la mesure où elle connaît une situation marginale à l'opposé des pays de vieille civilisation: les habitants ne cultivent ni le blé, ni la vigne, ils se nourrissent de poissons. Selon certains, ils vivent très âgés; d'autres les présentent comme des sauvages, tantôt bons, tantôt mauvais; l'imagination a pu se donner libre cours. Thulé est aussi remarquable pour ses volcans et leur activité, qui se manifestent d'une manière spectaculaire. Si les poètes et les auteurs de fiction ont peu utilisé le potentiel onirique de Thulé, une importance particulière à été donnée à un passage de la Médée de Sénèque qui a été considéré comme une prophétie et à laquelle Thulé est parfois mêlée, comme l'Atlantide ou la découverte de l'Amérique.

Dès le XVIII ${ }^{e}$ s., les connaissances géographiques ont beaucoup progressé - on en est à découvrir le Pacifique, - mais Thulé continue de susciter des questions et d'exciter l'imagination. Le plus souvent, Thulé est assimilée à l'Islande, mais on trouve aussi une île près de l'Estonie. L'explorateur Knud Rasmussen avait vécu au Groenland et fondé un comptoir dans la région afin de venir en aide aux Inuits. Il donna le nom de Thulé à un village des Inuits situé dans la baie de l'Etoile polaire, il en va de même pour son successeur Jean Malaurie. Beaucoup d'écrivains ont évoqué Thulé, dont Victor Hugo. Plus récemment, on retrouve Thulé mentionnée dans des livres pour enfants. Le nom apparaît maintenant jusque dans la publicité pour des voyages dans le Nord de l'Europe. L'A. évoque tous ces développements et analyse de manière critique les œuvres principales. On constate que, peu à peu, un glissement s'opère entre la Thulé située à l'extrémité du monde et une Thulé, accomplissement d'un rêve ou d'un idéal, 
qui conduit à une Thulé mythique, fruit de l'imagination, comme "Der König von Thulé » de Goethe, ballade mise en musique par plusieurs compositeurs, dont Schubert, Schumann et Liszt. Le poème sera traduit librement par Gérard de Nerval, il inspirera lui aussi des musiciens, comme Gounod et Berlioz.

6 Il s'agit d'une véritable somme, dont certains développements auraient peut-être pu être condensés. Le résumé qui précède, forcément bref, ne peut en donner qu'une idée. Cette somme est fondée sur des références bibliographiques très riches et illustrée de reproductions de plusieurs cartes de diverses époques. L'ouvrage se termine sur la liste chronologique des auteurs mentionnés, avec les éditions utilisées de leurs œuvres, une copieuse bibliographie, un index des noms d'hommes cités et un index des noms géographiques. Comme on le voit, l'A. s'est efforcée de donner aux chercheurs tous les instruments utiles et on ne peut que lui en être reconnaissant.

\section{AUTEURS}

JEAN-MICHEL RENAUD

Université de Liège 